\title{
Nail Involvement as a Predictor of Differential Treatment Effects of Secukinumab Versus Ustekinumab in Patients with Moderate to Severe Psoriasis
}

\author{
Curdin Conrad (D) - Christine-Elke Ortmann · Marc Vandemeulebroecke • \\ Torben Kasparek · Kristian Reich
}

Received: October 27, 2021 / Published online: December 6, 2021

(C) The Author(s) 2021

\begin{abstract}
Introduction: Patients with plaque psoriasis may experience varying levels of treatment response to different biologics, based on phenotypic characteristics and underlying genetic factors. Nail psoriasis is a common manifestation of psoriasis (approx. 50\% of patients) and has been linked to the human leukocyte antigen- $\mathrm{C}^{\star} 0602$ (HLA-C*0602) allele, which in turn has been associated with differential treatment responses to certain drugs. Here we investigate whether nail involvement in patients with psoriasis can predict differential skin responses to two biologics with different modes of action, namely secukinumab (anti-interleukin-17A) and ustekinumab (anti-interleukin-12/23), to ultimately guide treatment choice.
\end{abstract}

Methods: Data were pooled from the CLEAR and CLARITY studies and stratified post hoc by

C. Conrad $(\square)$

Department of Dermatology, Lausanne University

Hospital CHUV, Lausanne, Switzerland

e-mail: Curdin.Conrad@chuv.ch

C.-E. Ortmann · M. Vandemeulebroecke ·

T. Kasparek

Novartis Pharma AG, Basel, Switzerland

K. Reich

Translational Research in Inflammatory Skin

Disease, Institute for Health Services Research in

Dermatology and Nursing, University Medical

Center Hamburg-Eppendorf, Hamburg, Germany nail involvement status at baseline. Psoriasis Area and Severity Index (PASI) 75 and 90 responses over 52 weeks and absolute PASI $\leq 3$, $\leq 1$, and 0 values at weeks 16 and 52, were assessed.

Results: Based on the medical history, 30.4\% $(269 / 886)$ of the patients in the secukinumab arm and $29.7 \%(265 / 891)$ of patients in the ustekinumab arm presented with nail involvement. Nail involvement status had little to no impact on the efficacy of secukinumab, as comparable responses were achieved for patients with and without nail involvement in terms of PASI $75 / 90, \leq 3$, and 0 responses; slightly lower PASI $\leq 1$ reponses were achieved in patients with nail involvement. In the ustekinumab arm, patients with nail involvement achieved lower responses across all endpoints.

Conclusions: These findings indicate that nail involvement can serve as an observable prognostic factor for efficacy in skin psoriasis treatment and guide the choice between secukinumab and ustekinumab.

Trial Registration: CLEAR: NCT02074982; CLARITY: NCT02826603.

Keywords: Psoriasis;

Secukinumab; Ustekinumab; Nail involvement; Prognostic factor 


\section{Key Summary Points}

\section{Why carry out this study?}

Evidence in the literature suggests that human leukocyte antigen-C*0602 (HLA$\mathrm{C}^{\star} 0602$ )-negative patients with psoriasis are less responsive to certain treatment and that nail psoriasis is a common manifestation of psoriasis and is linked to the HLA-C*0602 allele.

Our aim was to determine whether nail involvement, which is associated with the HLA-C ${ }^{\star} 0602$ allele, might be used to predict therapy response with secukinumab and ustekinumab using the pooled data from the phase $3 \mathrm{~b}$ CLEAR and CLARITY trials.

\section{What has been learned from the study?}

Secukinumab demonstrated overall higher efficacy than ustekinumab in psoriasis and nail involvement status had no significant impact on the efficacy of secukinumab, as comparable responses were achieved for patients with and without nail involvement in terms of Psoriasis Area and Severity Index (PASI) 75/90, $\leq 3$, and 0 responses; patients with nail involvement achieved lower responses across all endpoints in the ustekinumab arm.

Nail involvement can serve as an observable indicator to predict treatment responses and could help to guide decision-making on the choice of future treatment with secukinumab or ustekinumab.

\section{INTRODUCTION}

Psoriasis is a chronic, T cell-mediated inflammatory skin disease. Plaque-type psoriasis, its most prevalent form, manifests as well-demarcated erythemato-squamous plaques on the skin [1]. The introduction of biologics for the therapeutic management of psoriasis has led to significant improvement in treatment responses. However, patients with psoriasis may respond differently to different biologics based on their phenotypic presentation, clinical characteristics, involvement areas, involved immune pathways and underlying genetic factors [2-4]. The human leukocyte antigen (HLA)$\mathrm{C} * 0602$ allele, which lies within the major histocompatibility complex (MHC) on chromosome 6 , has been established as a psoriasis risk allele [5]. It impacts the disease course, phenotypic features, disease severity, associated comorbidities and treatment response [2]. Previous studies have demonstrated that HLA$C^{\star} 0602$-negative patients are less responsive to methotrexate and ustekinumab $[2,6]$. In contrast, a phase $3 \mathrm{~b}$, multicentre, prospective study demonstrated that secukinumab achieved similar clinical responses in both HLA-C*0602positive and HLA-C*0602-negative patients, rendering determination of the HLA-C*0602 status unnecessary for secukinumab [7].

Nail psoriasis, a common manifestation of psoriasis, is associated with functional impairement, psychosocial disability and reduced quality of life. It occurs in up to $50 \%$ of patients with psoriaisis and in $70-80 \%$ of patients with psoriatic arthritis (PsA). It serves as a predictive factor for the development of PsA [8-10]. Interestingly, nail psoriasis is more common in HLA-C*0602-negative patients $[2,8]$.

Therefore, with the aim of improving outcomes in psoriasis patients, we assessed the hypothesis of whether nail involvement, with its negative association with the HLA-C*0602 allele, could act as an observable indicator to predict treatment response. This information could help to clinically stratify patients and provide informed treatment selection between the two commonly used biologics, secukinumab and ustekinumab. 


\section{METHODS}

\section{Study Design and Patients}

CLEAR and CLARITY were phase $3 \mathrm{~b}$ trials designed as 52-week, randomised, double-blind, active-controlled, parallel-group, superiority studies. In both studies, patients were randomised (1:1) to receive secukinumab $300 \mathrm{mg}$ or ustekinumab 45 or $90 \mathrm{mg}$ (according to body weight at baseline). Patients received secukinumab at baseline and weeks 1,2 , and 3, then every 4 weeks from weeks 4 to 48 , or ustekinumab at baseline and week 4 , and then every 12 weeks from weeks 16 to 40 . Patient eligibility criteria, primary and secondary objectives, assessments, statistical anlysis and results of both studies have been published [11, 12].

\section{Assessments}

Data from patients were pooled from both studies, and demographics and disease characteristics, including psoriasis severity, as measured by the Psoriasis Area and Severity Index (PASI), were assessed at baseline. Nail involvement in patients was identified from the case report form (CRF) that included information on ongoing and previous occurrence of nail psoriasis. Patients were included based on the nail involvement status declared in their medical history regardless of active nail involvement at study entry.

Efficacy in the pooled dataset was evaluated by the number (\%) of patients with reduction from baseline PASI $\geq 75 \%$ (PASI 75) and $\geq 90 \%$ (PASI 90), as well as the number (\%) of patients achieving an absolute (total score) PASI of $\leq 3$, $\leq 1$, and 0. PASI 75 and 90 scores were calculated over 52 weeks; $\leq 3, \leq 1$ and 0 values were assessed at weeks 16 and 52. Modified non-responder imputation was applied for analysis of PASI 75 and 90 outcomes. Analyses of PASI $\leq 3$, $\leq 1$ and 0 values were performed on observed data.

\section{Statistical Analysis}

Patients from the full analysis sets of the CLEAR and CLARITY studies were included. Categorical variables were expressed as counts and percentages. Continuous variables were presented as mean \pm standard deviation. As the current analyses were exploratory, no tests for statistical significance were performed. The 95\% confidence interval values are presented for all efficacy assessments.

\section{Ethics Compliance}

The CLEAR (NCT02074982) and CLARITY (NCT02826603) studies were conducted in accordance with the ethical principles of the Declaration of Helsinki at all the sites worldwide. Informed consent was obtained from all individual participants included in both studies. The protocols of both CLEAR (NCT02074982) and CLARITY (NCT02826603) studies were approved by the independent ethics committee/institutional review board of each center $[11,12]$.

\section{RESULTS}

Overall, 886 and 891 patients received secukinumab and ustekinumab, respectively; among whom $269(30.4 \%)$ and $265(29.7 \%)$ patients had a history of nail involvement in the secukinumab and ustekinumab arms, respectively. Of those with a history of nail involvement, nine and four patients in the secukinumab and ustekinumab arms, respectively, did not have ongoing nail psoriasis at study entry; for the subsequent analyses, these patients were included in the group with nail involvement. The baseline demographic and disease characteristics are shown in Table 1.

At baseline, patients with nail involvement had a longer disease duration (secukinumab 20.5 years; ustekinumab 17.3 years) than those without nail involvement (16.6 years in both arms). A higher proportion of patients with nail involvement had PsA (secukinumab 29.0\%; ustekinumab $24.2 \%$ ) than those without 
(secukinumab 16.5\%; ustekinumab 16.1\%) (Table 1).

Regardless of nail involvement, a higher percentage of secukinumab-treated patients versus those treated with ustekinumab attained PASI 75 and 90 responses over 52 weeks (Fig. 1). No significant impact of nail involvement was observed on the efficacy of secukinumab as a similar percentage of patients with and without nail involvement reached PASI 75 and 90 responses (Fig. 1). However, a higher proportion of ustekinumab-treated patients without nail involvement achieved PASI 75 and 90 scores than patients with nail involvement over 52 weeks (Fig. 1).

At weeks 16 and 52, secukinumab-treated patients showed higher $\mathrm{PASI} \leq 3$ responses compared with ustekinumab-treated patients, regardless of nail involvement (Fig. 2). Within the secukinumab arm, PASI $\leq 3$ responses were comparable in patients with (week 16: 87.2\%; week 52: 83.4\%) and without (week 16: 87.0\%; week 52: 86.0\%) nail involvement (Fig. 2). In the ustekinumab arm, higher PASI $\leq 3$ responses were observed in patients without nail involvement (week 16: 71.3\%; week 52: 75.8\%) than patients with nail involvement (week 16 : 64.6\%; week 52: 68.7\%) (Fig. 2). At weeks 16 and 52, differential efficacy was observed in PASI $\leq 1$ response; however, the difference between the groups with and without nail involvement was less pronounced in the secukinumab arm (Fig. 2). PASI 0 responses at weeks 16 and 52 are presented in Fig. 2.

Table 1 Baseline patient demographics and disease characteristics

\begin{tabular}{|c|c|c|c|c|c|c|}
\hline $\begin{array}{l}\text { Demographic/disease } \\
\text { characteristics }\end{array}$ & $\begin{array}{l}\text { SEC nails Y } \\
(n=269)^{\mathrm{a}}\end{array}$ & $\begin{array}{l}\text { SEC nails } N \\
(n=617)\end{array}$ & $\begin{array}{l}\text { SEC all } \\
(N=886)\end{array}$ & $\begin{array}{l}\text { UST nails Y } \\
(n=265)^{\mathrm{a}}\end{array}$ & $\begin{array}{l}\text { UST nails } N \\
(n=626)\end{array}$ & $\begin{array}{l}\text { UST all } \\
(N=891)\end{array}$ \\
\hline Age (years), mean $\pm S D$ & $45.4 \pm 13.3$ & $45.2 \pm 14.4$ & $45.3 \pm 14.0$ & $44.8 \pm 12.7$ & $45.1 \pm 14.5$ & $45.0 \pm 14.0$ \\
\hline Gender male, $n(\%)$ & $188(69.9)$ & $396(64.2)$ & $584(65.9)$ & $204(77.0)$ & $424(67.7)$ & $628(70.5)$ \\
\hline \multicolumn{7}{|l|}{ Race, $n(\%)$} \\
\hline Caucasian & $222(82.5)$ & $494(80.1)$ & $716(80.8)$ & $201(75.8)$ & $497(79.4)$ & $698(78.3)$ \\
\hline Black & $2(0.7)$ & $23(3.7)$ & $25(2.8)$ & $5(1.9)$ & $20(3.2)$ & $25(2.8)$ \\
\hline Asian & $32(11.9)$ & $51(8.3)$ & $83(9.4)$ & $45(17.0)$ & $51(8.1)$ & $96(10.8)$ \\
\hline Other & $13(4.9)$ & $49(7.9)$ & $62(7.0)$ & $14(5.3)$ & $58(9.3)$ & $72(8.1)$ \\
\hline Weight $(\mathrm{kg})$, mean $\pm S D$ & $90.2 \pm 23.5$ & $89.3 \pm 23.0$ & $89.6 \pm 23.2$ & $90.3 \pm 24.2$ & $90.9 \pm 23.9$ & $90.7 \pm 23.9$ \\
\hline Baseline PASI, mean \pm SD & $21.9 \pm 8.7$ & $20.8 \pm 8.8$ & $21.1 \pm 8.9$ & $21.7 \pm 8.5$ & $21.2 \pm 8.9$ & $21.4 \pm 8.8$ \\
\hline $\begin{array}{l}\text { Time since first } \mathrm{PsO} \\
\text { diagnosis (years), } \\
\text { mean } \pm \mathrm{SD}\end{array}$ & $20.5 \pm 12.9$ & $16.6 \pm 11.9$ & $17.8 \pm 12.3$ & $17.3 \pm 11.9$ & $16.6 \pm 12.8$ & $16.8 \pm 12.5$ \\
\hline Diagnosis of PsA, $n(\%)$ & $78(29.0)$ & $102(16.5)$ & $180(20.3)$ & $64(24.2)$ & $101(16.1)$ & $165(18.5)$ \\
\hline $\begin{array}{l}\text { Previous exposure to biologic } \\
\text { therapy, } n(\%)\end{array}$ & $48(17.8)$ & $109(17.7)$ & $157(17.7)$ & $55(20.8)$ & $120(19.2)$ & $175(19.6)$ \\
\hline
\end{tabular}

nails $Y$ Patients with nail involvement in medical history, nails $N$ patients without nail involvement in medical history, $N$ total number of evaluable patients, $n$ number of evaluable patients, PASI Psoriasis Area Severity Index, $P s A$ psoriatic arthritis, $P S O$ psoriasis, $S D$ standard deviation, SEC secukinumab, UST ustekinumab

${ }^{a}$ In the secukinumab group, 269 patients reported nail psoriasis in their medical history; for 9 patients it was not ongoing at study entry. In the ustekinumab group, 265 patients reported nail psoriasis in their medical history; for 4 patients it was not ongoing at study entry 


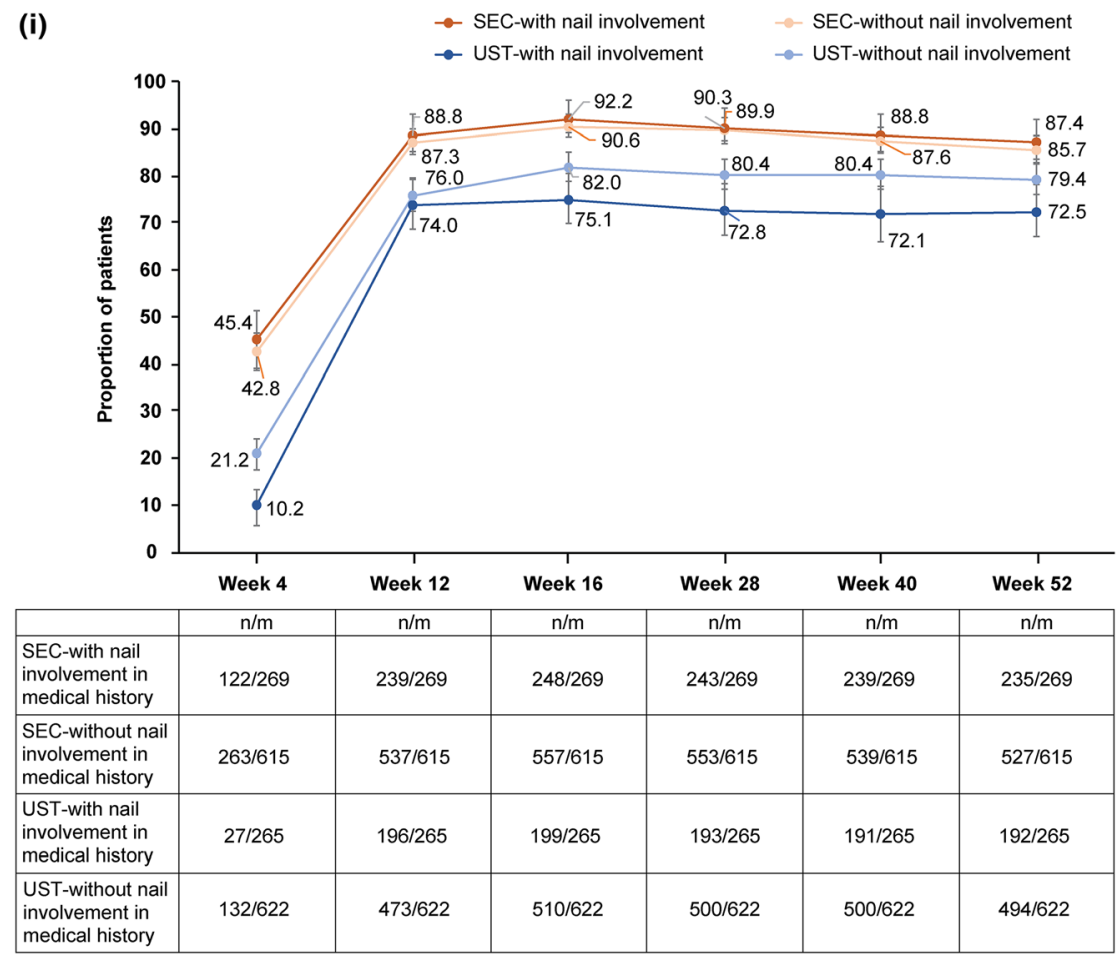

(ii) $\rightarrow$ SEC-with nail involvement $\rightarrow$ SEC-without nail involvement

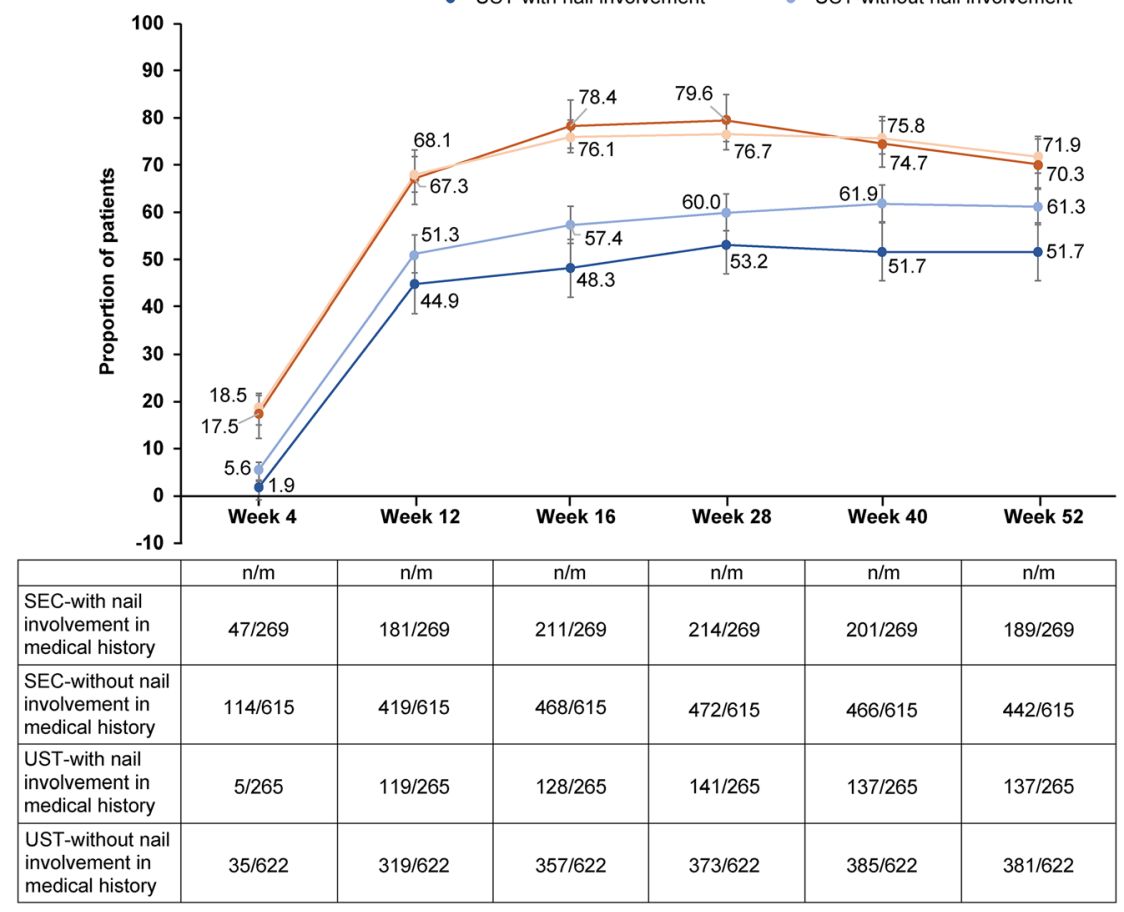

Fig. 1 Proportion of patients (95\% confidence interval) by treatment group and nail involvement status with PASI 75 (i) and PASI 90 (ii) response by visit (based on modified non-responder imputation). $m$ Total number of patients in the treatment arm, $n$ number of patients with response, PASI $75 / 90 \geq 75 \%$ and $\geq 90 \%$ reduction from baseline in Psoriasis Area and Severity Index score, SEC secukinumab, UST ustekinumab 


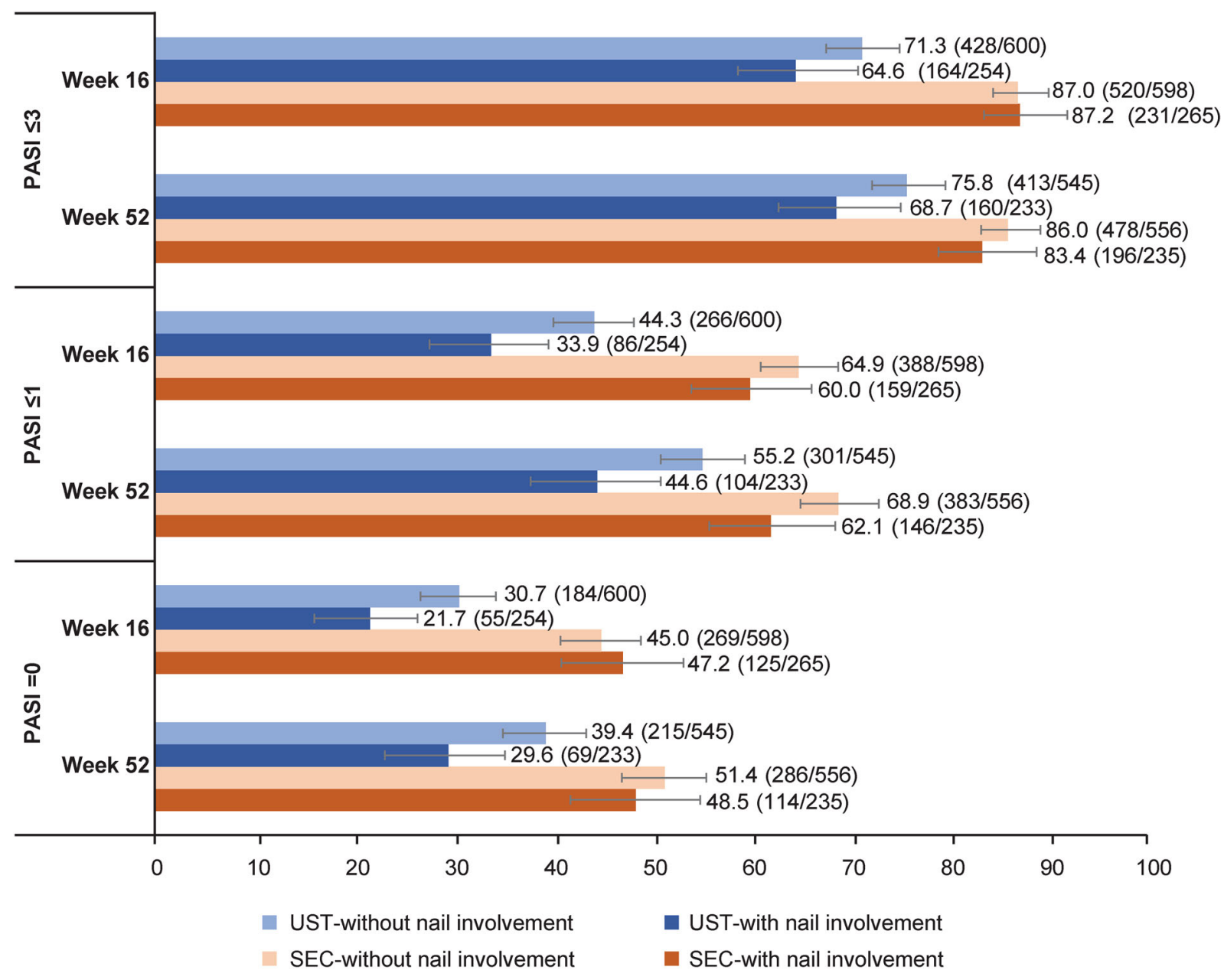

Fig. 2 Proportion of patients ( $95 \%$ confidence interval) by treatment group and nail involvement status with absolute (total score) PASI $\leq 3, \leq 1$ and 0 response by visit (based

\section{DISCUSSION}

In this study, we show that secukinumab demonstrated overall higher efficacy than ustekinumab in psoriasis and provided similar response rates in patients with and without nail involvement. However, among ustekinumabtreated patients, nail involvement was associated with reduced PASI responses, suggesting that nail psoriasis acts a predictor of inferior skin response to ustekinumab. Thus, when nail involvement was considered, secukinumab provided a disproportionately better clinical outcome than ustekinumab. These findings suggest that secukinumab should be favoured on observed data). PASI Psoriasis Area and Severity Index, SEC secukinumab, UST ustekinumab

for the treatment of skin psoriasis in patients with nail involvement.

HLA-C*0602 has previously been identified as a pharmacogenetic marker predicting superior response to ustekinumab in HLA-C ${ }^{*} 0602$ positive patients [6]. Nail psoriasis, however, is more frequent in HLA-C*0602-negative patients $[2,8]$. Accordingly, the finding that patients with nail involvement have a disproportionately inferior predicted outcome with ustekinumab compared to secukinumab might also be linked to the HLA-C*0602-status. This hypothesis is further supported by the fact that the HLA-C*0602-status, just like nail involvement, does not have an influence on the efficacy of secukinumab. 
The results of this study also suggest that the predominant pathogenic inflammatory pathways differ in psoriasis patients with and without nail involvement. One intriguing possibility is that early-onset psoriasis, which is associated with the HLA-C*0602 allele, is triggered by autoantigens that are presented to $\mathrm{T}$ cells in the context of the MHC-I HLA-C ${ }^{*} 0602$. Indeed, two autoantigens, LL37 and ADAMTS-like protein 5 (ADAMTSL5), have recently been shown to activate autoreactive CD8 $+\mathrm{T}$ cells in a HLA$\mathrm{C}^{\star} 0602$-dependent manner $[13,14]$. Moreover, intraepidermal CD8 $+\mathrm{T}$ cells producing interleukin (IL)-17, which have been identified as a key effector cells in psoriasis, are highly dependent on IL-23 $[15,16]$. Hence, the inhibition of the pathogenic $\mathrm{T}$ cell response via IL23 blockade could explain why ustekinumab shows an increased efficacy in HLA-C*0602positive patients and, in turn, in psoriasis patients without nail involvement. A recent study showed that activated $\mathrm{T}$ cells within the enthesis are able to secrete IL-17 without the need for additional IL-23 stimulation [17]. Considering similar pathogenic mechanisms in the skin, such as in the enthesis, this could explain the lower response rates of ustekinumab in patients with nail psoriasis.

The nail apparatus indeed represents a link between the skin disease and the joint involvement in psoriasis as it is an integral part of both the skin and the enthesis organ [18]. The association of nail psoriasis with PsA is well established and predicts future development of PsA [8-10]. IL-17 blockers represent the current gold standard in patients with both relevant skin and joint disease, as they have shown the same efficacy as anti-tumor necrosis factors (TNFs) in the treatment of PsA but were superior in achieving combined skin and joint improvements [19]. Based on indirect comparisons, ustekinumab is associated with lower response rates in PsA than TNF- and IL-17 blockers [20]. Therefore, nail involvement as a predictor for PsA could support the use of antiIL-17s based on their more robust efficacy in PsA. Moreover, we show here that nail psoriasis serves as an observable prognostic factor for treatment efficacy in skin psoriasis, suggesting that secukinumab should be preferred over ustekinumab in patients with nail involvement.

The current study is limited by its post hoc nature and the limitations intrinsic to any retrospective analysis. An additional limitation of the current analysis was that the assessment of severity and type of nail involvement and Nail Psoriasis Severity Index measurement was not performed in the study. Furthermore, the results with respect to the HLA-C*0602 allele should be interpreted with caution as HLA$C^{*} 0602$ status of patients was not assessed.

\section{CONCLUSION}

The results from the large dataset reported that nail involvement in psoriasis patients could act as an accessible and readily observable indicator to predict treatment responses. This information has clinically relevant implications and could facilitate clinical stratification and guide the treatment choice between secukinumab and ustekinumab.

\section{ACKNOWLEDGEMENTS}

The authors thank the participants of the study.

Funding. Sponsorship for this study and the Journal's Rapid Service Fee were funded by Novartis Pharma AG.

Medical Writing, Editorial, and Other Assistance. The authors thank Avinash Thakur and Shilpa Kakkar (Novartis Healthcare Pvt. Ltd, Hyderabad, India) for editorial and medical writing support, which was funded by Novartis Pharma AG, Basel, Switzerland, in accordance with the Good Publication Practice (GPP3) guidelines (http://www.ismpp.org/gpp3).

Authorship. All named authors meet the International Committee of Medical Journal Editors (ICMJE) criteria for authorship for this article, take responsibility for the integrity of the work as a whole, and have given their approval for this version to be published. 
Author Contributions. All authors contributed to manuscript concept and design. Data collection was done by Torben Kasparek. Christine-Elke Ortmann and Marc Vandemeulebroecke conducted the statistical analyses on the data. All authors interpreted the data, provided critical feedback on the manuscript, approved the final manuscript for submission and are accountable for the accuracy and integrity of the manuscript.

Prior Presentation. This study was presented in part at 29th European Academy of Dermatology and Venereology Virtual Congress, 29-31 October 2020.

Disclosures. Curdin Conrad has served as scientific adviser and/or clinical study investigator and/or paid speaker for AbbVie, Actelion, Almirall, Amgen, Boehringer Ingelheim, BristolMyers Squibb, Celgene, Galderma, Incyte, Janssen, LEO Pharma, Eli-Lilly, MSD, Novartis, Pfizer, Samsung and UCB. Christine-Elke Ortmann, Marc Vandemeulebroecke and Torben Kasparek are employees of Novartis Pharma AG, Basel, Switzerland. Kristian Reich has served as advisor and/or paid speaker for and/or participated in clinical trials sponsored by Abbvie, Affibody, Almirall, Amgen, Avillion, Biogen, Boehringer Ingelheim, Bristol-Myers Squibb, Celgene, Centocor, Covagen, Dermira, Forward Pharma, Fresenius Medical Care, Galapagos, GlaxoSmithKline, Janssen-Cilag, Kyowa Kirin, Leo, Eli-Lilly, Medac, Merck Sharp \& Dohme, Novartis, Miltenyi Biotec, Ocean Pharma, Pfizer, Regeneron, Samsung Bioepis, Sanofi, Sun Pharma, Takeda, UCB, Valeant and Xenoport.

Compliance with Ethics Guidelines. The CLEAR (NCT02074982) and CLARITY (NCT02826603) studies were conducted in accordance with the ethical principles of the Declaration of Helsinki at all the sites worldwide. Informed consent was obtained from all individual participants included in both studies. The protocols of both CLEAR (NCT02074982) and CLARITY (NCT02826603) studies were approved by the independent ethics committee/institutional review board of each center $[11,12]$
Data Availability. The datasets generated and/or analysed during the current study are not publicly available. Novartis is committed to sharing with qualified external researchers access to patient-level data and supporting clinical documents from eligible studies. These requests are reviewed and approved on the basis of scientific merit. All data provided are anonymised to respect the privacy of patients who have participated in the trial in line with applicable laws and regulations. The data may be requested from the corresponding author of the manuscript.

Open Access. This article is licensed under a Creative Commons Attribution-NonCommercial 4.0 International License, which permits any non-commercial use, sharing, adaptation, distribution and reproduction in any medium or format, as long as you give appropriate credit to the original author(s) and the source, provide a link to the Creative Commons licence, and indicate if changes were made. The images or other third party material in this article are included in the article's Creative Commons licence, unless indicated otherwise in a credit line to the material. If material is not included in the article's Creative Commons licence and your intended use is not permitted by statutory regulation or exceeds the permitted use, you will need to obtain permission directly from the copyright holder. To view a copy of this licence, visit http://creativecommons.org/licenses/bync/4.0/.

\section{REFERENCES}

1. Conrad C, Gilliet M. Psoriasis: from pathogenesis to targeted therapies. Clin Rev Allergy Immunol. 2018;54:102-13.

2. Chen L, Tsai TF. HLA-Cw6 and psoriasis. Br J Dermatol. 2018;178:854-62.

3. Edson-Heredia E, Sterling KL, Alatorre CI, et al. Heterogeneity of response to biologic treatment: perspective for psoriasis. J Invest Dermatol. 2014;134:18-23. 
4. Jiménez CM, Pérez Ramírez C, Sánchez Martín A, et al. Influence of genetic polymorphisms on response to biologics in moderate-to-severe psoriasis. J Pers Med. 2021;11:293.

5. Gudjonsson JE, Karason A, Antonsdottir A, et al. Psoriasis patients who are homozygous for the HLA$\mathrm{CW}^{*} 0602$ allele have a 2.5 -fold increased risk of developing psoriasis compared with Cw6 heterozygotes. Br J Dermatol. 2003;148:233-5.

6. Talamonti M, Botti E, Galluzzo M, et al. Pharmacogenetics of psoriasis: HLA-Cw6 but not LCE3B/3C deletion nor TNFAIP3 polymorphism predisposes to clinical response to interleukin $12 / 23$ blocker ustekinumab. Br J Dermatol. 2013;169:458-63.

7. Costanzo A, Bianchi L, Flori ML, et al. Secukinumab shows high efficacy irrespective of HLA-Cw6 status in patients with moderate-to-severe plaque-type psoriasis: SUPREME study. Br J Dermatol. 2018;179: 1072-80.

8. Gudjónsson JE, Kárason A, Antonsdóttir AA, et al. HLA-Cw6-positive and HLA-Cw6-negative patients with psoriasis vulgaris have distinct clinical features. J Invest Dermatol. 2002;118:362-5.

9. Reich K. Approach to managing patients with nail psoriasis. J Eur Acad Dermatol Venereol. 2009;23(Suppl 1):15-21.

10. Wilson FC, Icen M, Crowson CS, McEvoy MT, Gabriel SE, Kremers HM. Incidence and clinical predictors of psoriatic arthritis in patients with psoriasis: a population-based study. Arthritis Rheum. 2009;61:233-9.

11. Bagel J, Nia J, Hashim PW, et al. Secukinumab is superior to ustekinumab in clearing skin in patients with moderate to severe plaque psoriasis (16-week CLARITY results). Dermatol Ther (Heidelb). 2018;8: 571-9.

12. Thaçi D, Blauvelt A, Reich K, et al. Secukinumab is superior to ustekinumab in clearing skin of subjects with moderate to severe plaque psoriasis: CLEAR, a randomized controlled trial. J Am Acad Dermatol. 2015;73:400-9.

13. Arakawa A, Siewert K, Stöhr J, et al. Melanocyte antigen triggers autoimmunity in human psoriasis. J Exp Med. 2015;212:2203-12.

14. Lande R, Botti E, Jandus C, et al. The antimicrobial peptide LL37 is a T-cell autoantigen in psoriasis. Nat Commun. 2014;5:5621.

15. Di Meglio P, Villanova F, Navarini AA, et al. Targeting CD8(+) $\mathrm{T}$ cells prevents psoriasis development. J Allergy Clin Immunol. 2016;138:274-6.e6.

16. Ciric B, El-behi M, Cabrera R, Zhang G-X, Rostami A. IL-23 Drives pathogenic IL-17-producing CD8+T cells. J Immunol. 2009;182:5296-305.

17. Watad A, Rowe H, Russell T, et al. Normal human enthesis harbours conventional CD4+ and CD8+ T cells with regulatory features and inducible IL-17A and TNF expression. Ann Rheum Dis. 2020;79: 1044-54.

18. Tan AL, Benjamin M, Toumi $\mathrm{H}$, et al. The relationship between the extensor tendon enthesis and the nail in distal interphalangeal joint disease in psoriatic arthritis-a high-resolution MRI and histological study. Rheumatology (Oxford). 2007;46: 253-6.

19. McInnes IB, Behrens F, Mease PJ, et al. Secukinumab versus adalimumab for treatment of active psoriatic arthritis (EXCEED): a double-blind, parallel-group, randomised, active-controlled, phase $3 \mathrm{~b}$ trial. Lancet. 2020;395:1496-505.

20. Menter A, Krueger GG, Paek SY, Kivelevitch D, Adamopoulos IE, Langley RG. Interleukin-17 and interleukin-23: a narrative review of mechanisms of action in psoriasis and associated comorbidities. Dermatol Ther (Heidelb). 2021;11:385-400. 